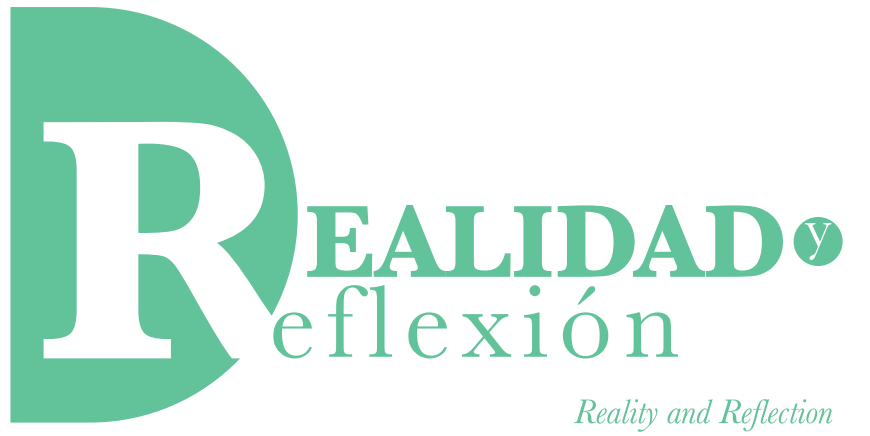

ISSN 1992-6510

Año 20, N 51, San Salvador, El Salvador, Centroamérica. Revista Semestral Enero-Junio 2020

YEAR 20, $N^{\circ}$ 51, SAN SALVADOR, EL SALVADOR, CENTRAL AMERICA. SEMESTRAL JOURNAL JANUARY-JuNE 2020

\title{
Centroamérica y el cambio climático: De la planificación a la acción
}

\section{Central America and climate change: From planning to action}

Miguel Antonio Lazo Vega Lic. Computación por la Universidad Capitán General Gerardo Barrios, El Salvador.

Máster en Logística Integral por Bureau Veritas Business School, España. Máster en Comercio Internacional por el Instituto Superior de Educación, Administración y Desarrollo asociado al Centro Universitario Villanueva adscrito a la

Universidad Complutense de Madrid, España. Doctor en Ciencias Económicas y Administrativas por la Universidad para la Cooperación Internacional, Costa Rica. mikelazo@hotmail.com,mlazo@sigmaq.com

DOI: $10.5377 /$ ryr.v51i0.9845 


\section{RESUMEN}

El principal objetivo de este artículo ha sido evaluar importantes hallazgos relacionados al cambio climático en la región centroamericana y en América Latina y el Caribe; para ello, se hace un análisis de estudios previos llevados a cabo por organismos regionales e internacionales implicados en la lucha contra el cambio climático y que se complementan con el punto de vista en relación al tema del autor. Haciendo uso de este análisis el artículo presenta los siguientes elementos: 1) Una visión del nuevo mundo que se tiene por los efectos relacionados al cambio climático; 2) Una serie de impactos en la región centroamericana provocados por el cambio climático en los últimos años; 3) El crecimiento económico de la región y su relación con el cambio climático, 4) Un breve detalle de las acciones y estrategias llevadas a cabo a nivel mundial y regional en relación al cambio climático, y 5) Finalmente, se plantean algunas conclusiones y recomendaciones que permitirá la consolidación de conocimientos de ésta investigación..

Palabras claves: América Central, cambio climático, impactos, estrategias.

\section{ABSTRACT}

The main goal of this article has been to assess important findings about climate change in Central America and Latin America and the Caribbean; for achieve this, the article includes an analysis of previous researches developed by regionals and internationals organizations involved in the fight against climate change and which are complemented with the author's point of view. Within this analysis, the article includes the following elements: 1) A vision of a new world for the effects of climate change; 2) A series of impacts in America Central for the climate change in last years; 3) The economic growth and its relationship with climate change in the region, 4) A brief description of the actions and strategies carried out in the world and the region about climate change, and 5) Finally, some reflections and recommendations are proposed which should allow the consolidation of knowledges about this research.

Keywords: Central America, climate change, impacts, strategies. 


\section{Introducción}

El cambio climático ha sido considerado por muchos especialistas, investigadores, líderes mundiales y religiosos, organismos regionales e internacionales, figuras públicas, entre muchos otros actores, como uno de los más grandes problemas y desafíos de nuestra era debido a las graves consecuencias asociadas a él; consecuencias que a estas alturas en muchas regiones ya son palpables al estar en el frente de batalla y que le han brindado prácticamente un ultimátum a la humanidad sobre lo que está por venir si no se trata de cambiar el rumbo que se lleva; vale mencionar y de diferenciar antes de profundizar en esta temática, que el clima del planeta Tierra siempre ha presentado variaciones, desde su origen y en todo su proceso evolutivo, fácilmente puede decirse que es un fenómeno natural asociado; sin embargo, la investigación científica sugiere que desde la Revolución Industrial $^{1}$ y por el impacto significativo de las actividades llevadas a cabo por los seres humanos desde entonces, se ha acelerado el aumento de la temperatura en la atmósfera del planeta, lo que ha provocado cambios sin precedentes en el sistema climático mundial.

Lo crítico de este escenario es que presume que cualquier modificación en el clima conducirá a la desestabilización de las condiciones ambientales y sociales en todo el planeta, dicho en otras palabras, se pone en peligro con su presencia la conservación de los ecosistemas naturales y la

1 Serie de transformaciones económicas, políticas, sociales, etc. que se iniciaron en Inglaterra en el siglo XVIII y que se expandió hacia los demás países de la Europa Continental que tenía como denominador común la mecanización de la industria; a tal punto que se llegó a catalogar la misma como el paso de una economía de herramienta a una economía de máquina (Silva y Mata de Grossi, 2005). sustentabilidad de los sistemas socioeconómicos (Colette, 2005); con relación a este último punto, todos los países y sus gobiernos tienen la meta marcada en piedra de buscar siempre el anhelado crecimiento económico y el bienestar social de sus habitantes; situación que ha traído consigo en la mayor parte de los casos, impactos significativos y negativos en el medio ambiente.

Este escenario no es ajeno a la región de América Latina y el Caribe, y específicamente en Centroamérica como tal; más aún si se considera que su ubicación geográfica la coloca en un riesgo latente a fenómenos naturales de diversa índole; a lo cual se le adiciona su delicada situación económica y social y que han llevado a catalogar a la región como altamente vulnerable ante los efectos del cambio climático, ocasionado en gran medida por el tremendo daño que como región se ha hecho a su diverso ecosistema por la demanda creciente de recursos naturales, la expansión urbana sin regulación, el desordenado proceso de asentamientos urbanos y las actividades productivas tales como la extracción de recursos con poco o ningún control, llevando en muchos de esos casos a una pérdida irreversible o a una degradación pronunciada y sostenida de tales recursos; situación crítica que ha sido percibida por muchas entidades, organismos y gobiernos de sus distintos países, quienes desde hace varias décadas se han dado a la tarea de preparar estrategias y mecanismos de acción y adaptación que traten de cambiar dicha situación a nivel mundial y en la región como tal; uno de los principales frutos a nivel mundial de este esfuerzo nace en el año de 1988, cuando el Programa de las Naciones Unidas para el Medio Ambiente 
$\left(\right.$ PNUMA $\left.^{2}\right)$ y la Organización Meteorológica Mundial $\left(\mathrm{OMM}^{3}\right)$ establecen el Grupo Intergubernamental de Expertos sobre el Cambio Climático (Reconocido por sus siglas en inglés, IPCC, 1 de mayo de 2015) con la finalidad de proporcionar información objetiva, clara, equilibrada y neutral del estado actual de conocimientos sobre el cambio climático a los responsables políticos y otros sectores interesados; vale indicar que desde su creación, IPCC ha lanzado una serie de documentos técnicos, informes especiales e informes de evaluación que están abiertos al público; claramente lo que se busca con este cúmulo de conocimientos es que los países y regiones logren una transición a economías ambientalmente más sostenibles que permitan la supervivencia del ser humano, mediante la inclusión y logro de los Objetivos de Desarrollo Sostenible ${ }^{4}$ (ODS) planteados por las Naciones Unidas (Comisión Económica para América Latina y el Caribe (CEPAL), 2015a) en la Agenda $2030^{5}$ para el Desarrollo Sostenible; presta especial atención un informe

2 E1 PNUMA es el portavoz del medio ambiente dentro del sistema de las Naciones Unidas y promueve el uso racional y el desarrollo sostenible del medio ambiente mundial (PNUMA, s.f.).

3 La OMM es el portavoz autorizado del sistema de las Naciones Unidas sobre el estado y el comportamiento de la atmósfera de la Tierra, su interacción con la tierra y los océanos, el tiempo y el clima que genera, y la consiguiente distribución de los recursos hídricos (OMM, s.f.)

4 Los ODS son el plan maestro para conseguir un futuro sostenible para todos; los cuales se interrelacionan entre sí e incorporan los desafíos globales a los que nos enfrentamos día a día, como la pobreza, la desigualdad, el clima, la degradación ambiental, la prosperidad, la paz y la justicia; los mismos se gestaron en la Conferencia de las Naciones Unidas de Río de Janeiro en 2012 y entraron en vigor en 2016 (Programa de las Naciones Unidas (PNUD), 2016).

5 La Agenda 2030 es un plan de acción mundial que proporciona un modelo para una prosperidad compartida en un mundo sostenible que está conformado por los 17 ODS. reciente de las Naciones Unidas (2019) donde se manifiesta tajantemente que el área que requiere medidas sustanciales más urgentes en la actualidad es la del cambio climático.

\section{El nuevo mundo a causa del cambio climático}

El cambio climático se ha convertido en uno de los desafíos más grandes del planeta debido en gran medida a la mano del hombre, quien en su afán por mantener su estilo de vida y desarrollo insostenible ha contribuido de manera significativa al aumento de las temperaturas en todo el mundo, al alza en el nivel del mar que afecta a regiones y países enteros, a la contaminación atmosférica por medio del aumento de los gases que provocan el efecto invernadero $\left(\mathrm{GEI}^{6}\right)$ como lo es el dióxido de carbono $^{7}\left(\mathrm{CO}_{2}\right)$ y el metano ${ }^{8}\left(\mathrm{CH}_{4}\right)$, al deterioro significativo de sus recursos naturales como lo es el agua, a la tala indiscriminada de sus principales bosques tropicales que son los pulmones del planeta, al acelerado descongelamiento de grandes glaciares y que están llegando a un punto de no retorno (CEPAL, 2015b), entre otros graves problemas; todo este escenario ha afectado la forma de vida de millones de personas y a cientos de miles de especies a nivel mundial y ha planteado serios lineamientos sobre lo que será un nuevo

6 Los GEI son aquellos gases presentes en la atmósfera que contribuyen al efecto invernadero; entendiendo por efecto invernadero, el proceso por el que la radiación térmica emitida por la atmósfera es absorbida por los gases presentes e irradiada en todas las direcciones; dicha emisión continuada provoca un mayor calentamiento de la superficie terrestre (Compañía Española de Petróleos (CEPSA), 2015).

7 El dióxido de carbono, es un gas incoloro, denso y poco reactivo, que forma parte de la capa de la atmósfera más cercana a la Tierra.

8 El metano es producto final de la fermentación que sufren los alimentos en el rumen, que en términos de energía constituye una pérdida y en términos ambientales contribuye al cambio climático. 


\section{a) Anomalías de temperaturas anuales, 1880-2019}

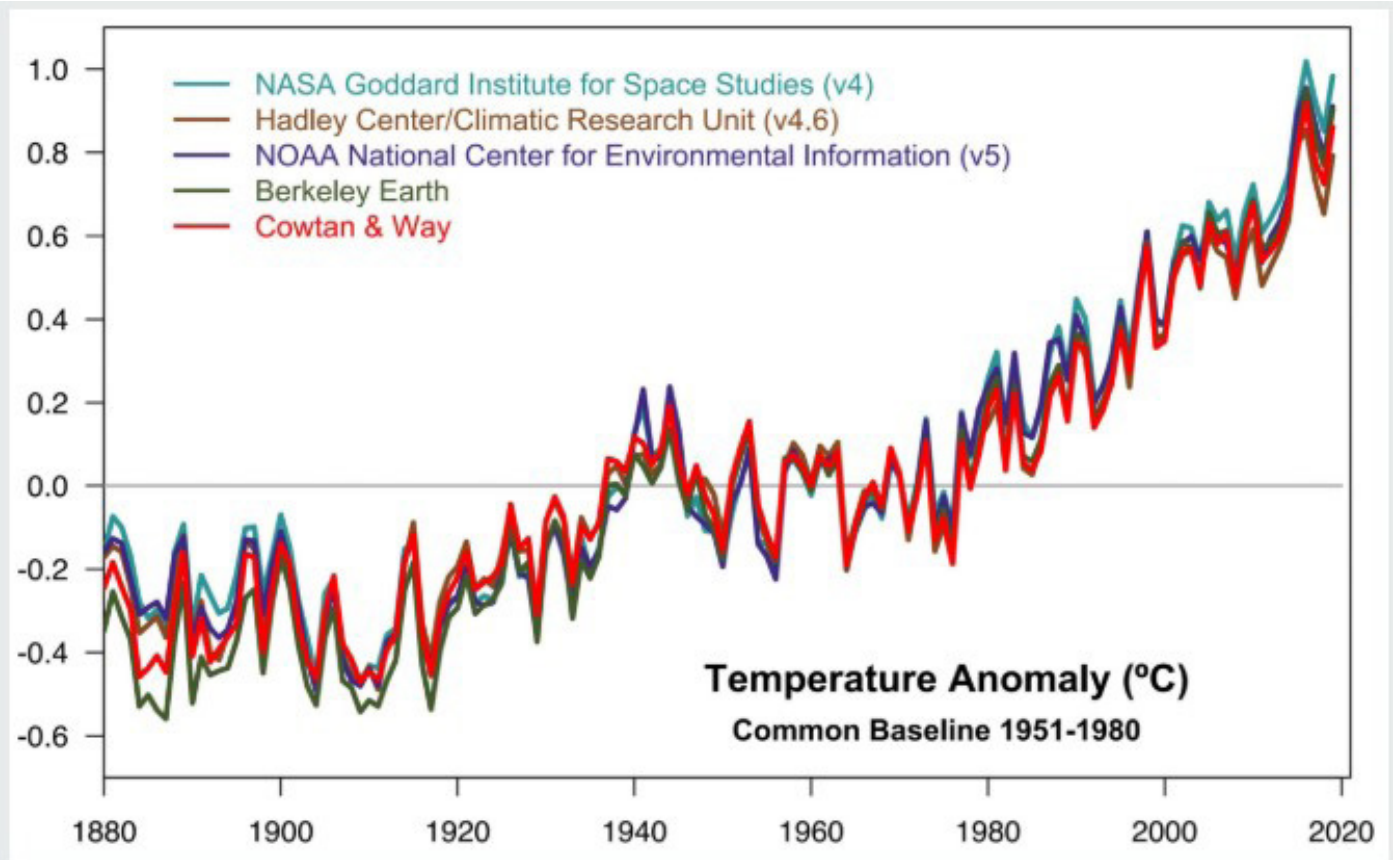

Figura 1. a) Anomalías de temperaturas anuales, 1880-2019. Gráfico que muestra anomalías de temperatura anuales desde 1880 hasta 2019, con respecto a la media de 1951-1980; según los datos registrados por la NASA (National Aeronautics and Space Administration), NOAA (National Oceanic and Atmospheric Administration), el grupo de investigación de Berkeley Earth, el Met Office Hadley Centre (Reino Unido) y el análisis Cowtan and Way; aunque hay pequeñas variaciones de un año a otro, los cinco registros de temperatura muestran picos y valles sincronizados entre sí. Todos indican un rápido calentamiento en las últimas décadas, y todos señalan que la última década ha sido la más cálida en el registro.

Fuente: NASA GISS / Gavin Schmid, a través de herramienta GISTEMP v4.

Nota: GISS es un laboratorio integrado en la División de Ciencias de la Tierra del Centro de Vuelo Espacial Goddard de la NASA en Greenbelt, Maryland.)

mundo en éstas condiciones. Vale mencionar que existe suficiente evidencia científica que documenta el problema y que plantea que el cambio climático ha sido ocasionado por un modelo de desarrollo de visión a corto plazo, pero principalmente insostenible basado en la degradación, contaminación y extracción indiscriminada de los recursos naturales a nivel mundial; así como a una creciente dependencia de los combustibles fósiles, entre otros eventos que han profundizado el problema; en este apartado es necesario hacer mención de los GEI y su participación significativa del fenómeno, ya que se considera que el exceso de dichas emisiones -se tuvo un incremento significativo del $50 \%$ en las emisiones desde 1990- ha contribuido en el aumento de la temperatura media de la Tierra que aumentó $0.85^{\circ} \mathrm{C}$ entre 1880 y 2012 (IPCC, 2014); 
por tal razón es que las Naciones Unidas en sus ODS ha puesto especial énfasis en reducir éstas, ya que se considera que de no mermar las mismas, fácilmente se puede aumentar la temperatura en $3^{\circ} \mathrm{C}$ o incluso más en algunas zonas del planeta (CEPAL, 2018).

Por otra parte, se tiene la particularidad que los países desarrollados son considerados los principales contaminantes e impulsadores de éste grave fenómeno, pero son los que se ven menos afectados en sus inicios gracias a su capacidad de gestión, adaptación y recursos; caso contrario a lo que sucede en países en vías de desarrollo, los cuales no tienen una capacidad rápida de respuesta y de adaptación, y que por ende, son los que han soportado inicialmente la carga y embate de éstos fenómenos y que serán los principales perjudicados inicialmente de éste escenario; esto de igual manera, le brinda a los países desarrollados una responsabilidad "histórica" sobre los cambios que se están dando y por la consecuente agudización de la situación de vulnerabilidad que ha puesto en riesgo las generaciones actuales y futuras, incluidos sus propios beneficios y condiciones privilegiadas con las que viven actualmente (Comisión Centroamericana de Ambiente y Desarrollo (CECAD) - Sistema de Integración Centroamericana (SICA), 2010).

\section{b) Emisiones globales de $\mathrm{CO}_{2}, 2019$.}

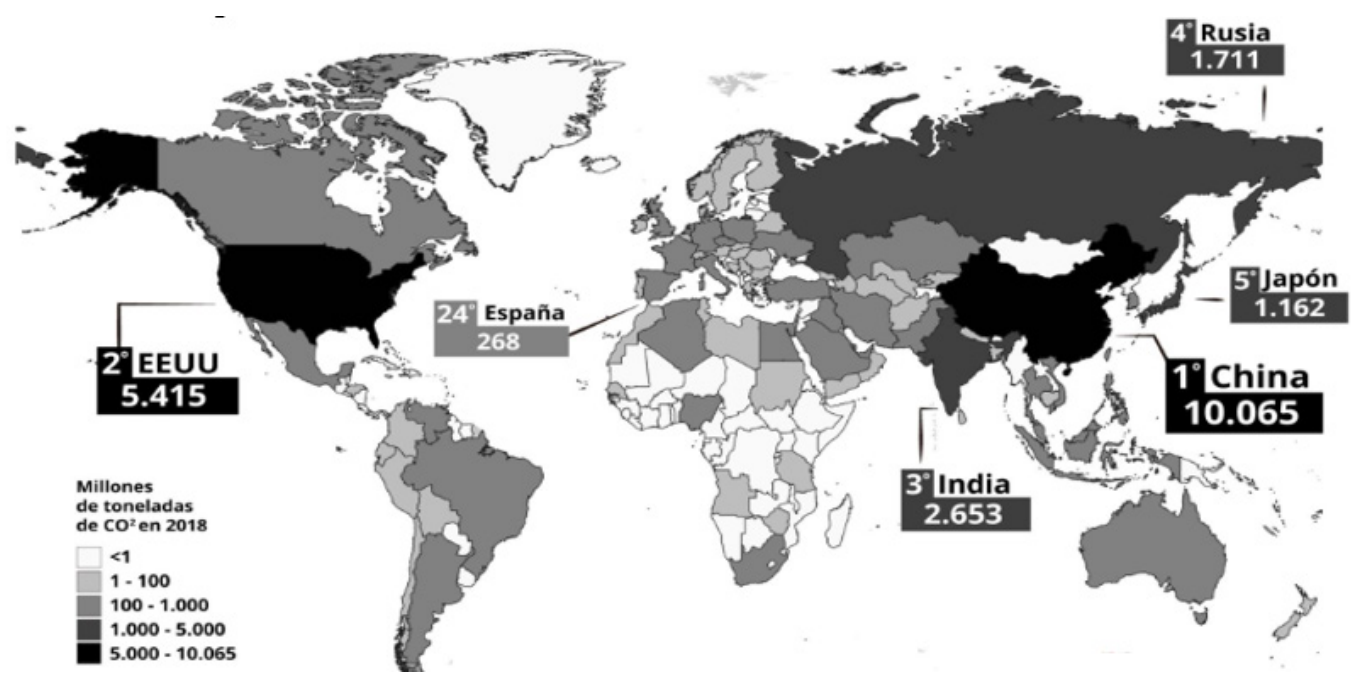

Figura 2. b) Emisiones globales de CO2, 2018. Mapa que muestra los niveles alcanzados en millones de toneladas de $\mathrm{CO}_{2}$ en el año 2018, período en el que los niveles llegaron a 407.8 partes por millón (ppm) frente a los 405.5 ppm de 2017 según datos de la OMM (2019) y que constituyeron un nuevo récord.

Fuente: Global Carbon Project, a través de herramienta Global Carbon Atlas.

Nota: Las ppm es la unidad empleada de forma habitual para indicar la existencia de elementos en muy pequeña cantidad, lo que se conoce como traza en una mezcla, concretamente, aire. 


\section{c) Emisiones antropógenas anuales totales de GEI por gases, 1970-2010.}

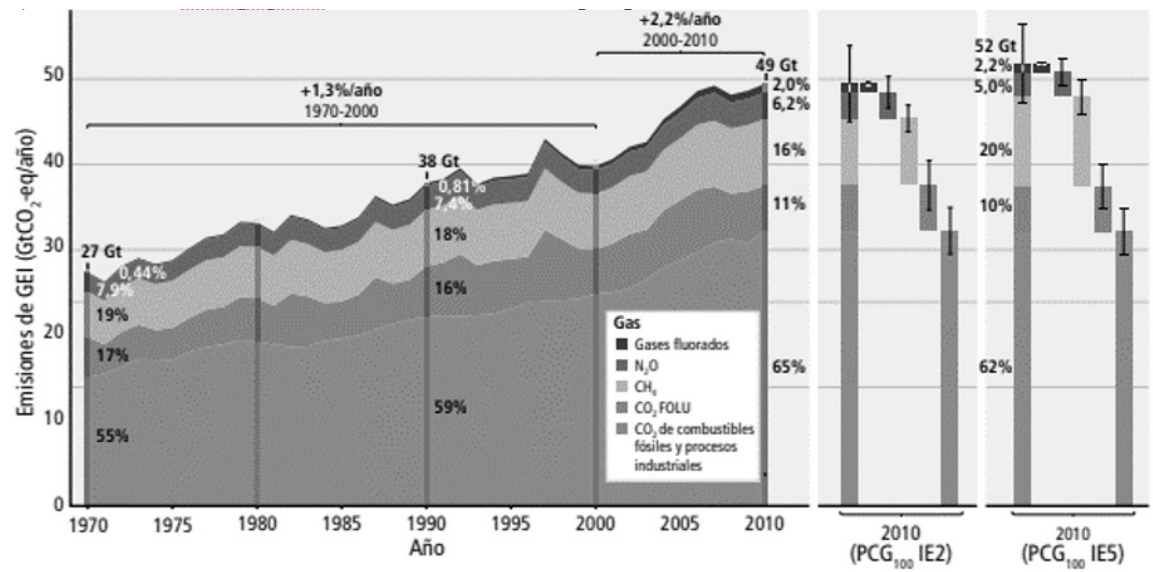

Figura 3. c) Emisiones antropógenas anuales totales de gases de efecto invernadero (GEI) (gigatonelada de $\mathrm{CO}_{2}$-equivalente al año, $\mathrm{GtCO}_{2}$-eq/año), 1970 y 2010, por gases: $\mathrm{CO}_{2}$ procedente de la quema de combustibles fósiles y procesos industriales; $\mathrm{CO}_{2}$ procedente de la silvicultura y otros usos del suelo (FOLU); metano $\left(\mathrm{CH}_{4}\right)$; óxido nitroso $\left(\mathrm{N}_{2} \mathrm{O}\right)$; gases fluorados abarcados en el Protocolo de Kyoto. A la derecha se muestran las emisiones de 2010, con ponderaciones de emisiones de $\mathrm{CO}_{2}$-equivalente basadas en valores de los Informes de Evaluación Segundo y Quinto del IPCC.

\section{Impactos del cambio climático en la región centroamericana}

$\mathrm{El}$ istmo centroamericano ${ }^{9}$ es una de las regiones más vulnerables a los efectos del cambio climático debido a su ubicación geográfica que la hace proclive a fenómenos naturales diversos, por un lado se forma un delgado puente que conecta a América del Norte con América del Sur, y se cuenta con costas en el Océano Pacífico y Atlántico, así como con cadenas montañosas que forman parte de su relieve; también buena parte de su territorio es de baja altura y esta sobre el nivel del mar; esto hace que la región cuente con una diversidad de ambientes y ecosistemas marinos ${ }^{10}$

9 Conjunto ecosistémico único que abarca desde el sur de México hasta la frontera panameña-colombiana compuesta por siete países: Panamá, Costa, Rica, Nicaragua, Honduras, E1 Salvador, Guatemala y Belice.

10 La región cuenta con playas, dunas, acantilados, franjas litorales rocosas, lagunas arrecifales y costeras, humedales, selvas, entre muchos otros. producto de su proceso de formación hace más de 3.5 millones de años (Fundación Heinrich Böll, 2018) y que exista una variabilidad climática que permite la presencia de fenómenos tales como sequías ${ }^{11}$, inundaciones, lluvias, deslizamientos, entre muchos otros; sin duda, esto trae consigo una serie de problemas para sus más de 40 millones de habitantes según datos oficiales del Banco Mundial, los cuales se ven afectados por estos escenarios; y más si consideramos que la región en sí, depende en gran medida de la agricultura como fuente de alimentos, y que indudablemente pone en riesgo la seguridad alimentaria que se tiene.

11 La OMM define a la sequía como un fenómeno perjudicial que se produce a raíz de niveles de precipitación inferiores a lo esperado o normal y que, cuando se prolonga durante una estación o períodos más largos, hace que las precipitaciones sean insuficientes para responder a las demandas de la sociedad y del medio ambiente (2006). 


\section{d) Panorama de la región centroamericana.}

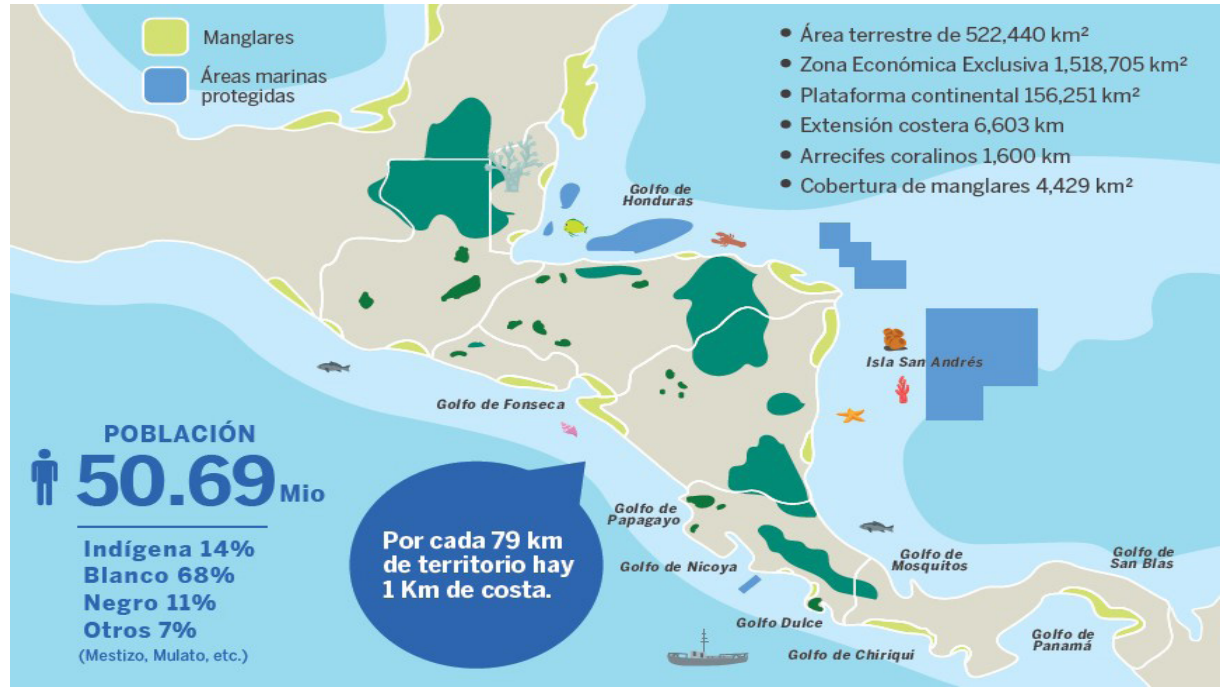

Figura 4. Panorama de la región centroamericana, donde se visualizan los manglares y las áreas marinas protegidas; así como la distribución de las zonas que conforman la región. Fuentes: Informe de "Atlas de los Océanos. Adenda Centroamérica" 2018 de la Fundación Heinrich Böll.

\section{Gráfico 1}

Población de los países de Centroamérica, 2018

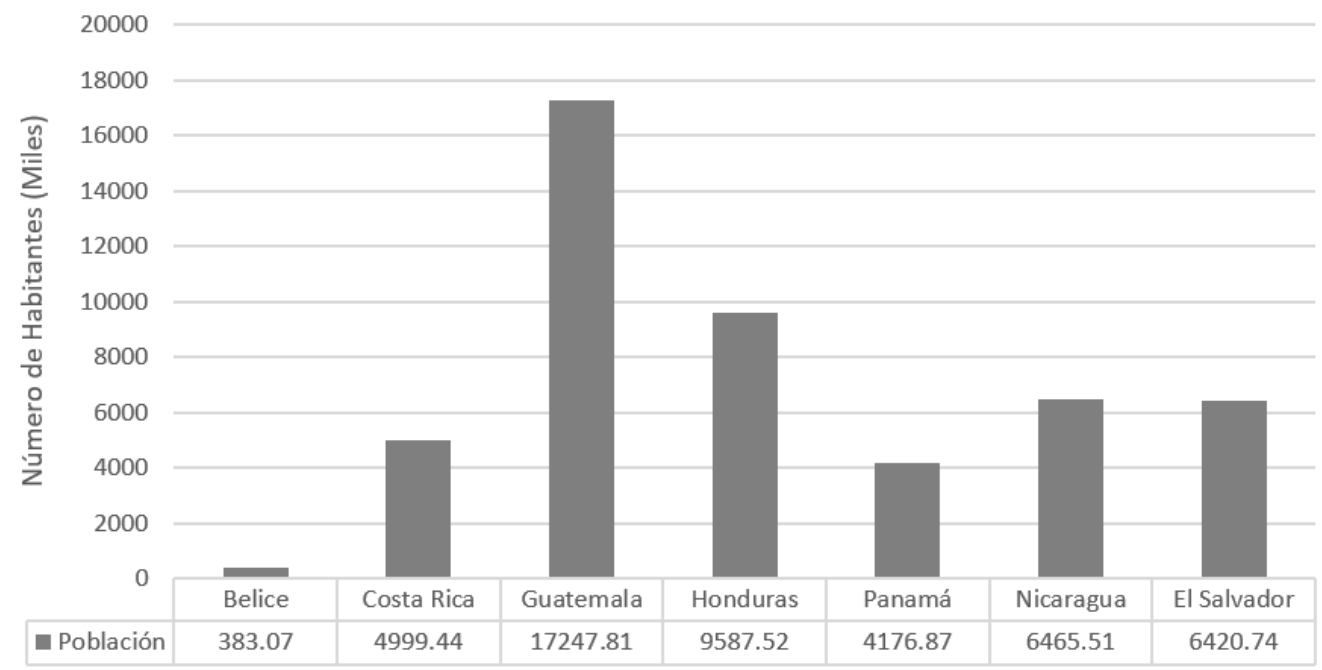

Fuente: Elaboración propia del autor con datos obtenidos del Banco Mundial. 
La situación de la región como tal y de sus países con relación a los efectos del cambio climático también es visible en el famoso Índice de Riesgo Climático Global (IRC ${ }^{12}$ ), donde salen a relucir los países de la región, específicamente Honduras y Nicaragua que en el período comprendido entre 1998 y 2017 se encuentran en el top ten de los países más afectados por eventos climáticos extremos; y en donde otros países de la región como El Salvador, ocuparon el primer lugar de dicha clasificación en períodos anteriores; cabe resaltar que en el período indicado murieron más de 526,000 personas como consecuencia directa de más de 11,500 fenómenos meteorológicos extremos y se generaron pérdidas de aproximadamente 3.47 billones de dólares estadounidenses (Germanwatch, 2019a).

Esta situación de riesgo que se indica, se ha visto reflejada en mayor medida en los últimos años; ya que independientemente de que en la región exista una variabilidad climática en diversas escalas de tiempo, la misma se ha presentado en mayor intensidad, duración y frecuencia; rompiendo en muchos casos récord históricos de tales eventos en la región (Evangelista, 2018); vale mencionar que aunque las pérdidas monetarias sean más altas en los países ricos por culpa de éstos eventos; la pérdida de vidas, la emergencia humana y la amenaza existencial es mucho más extendida

12 El IRC es un análisis basado en un conjunto de datos fiables sobre los impactos de los eventos climáticos extremos y de los datos socioeconómicos asociados a ellos; sin embargo, vale recalcar que el mismo no debe de confundirse con un sistema de clasificación completo de la vulnerabilidad climática; de igual manera, indica el nivel de exposición y la vulnerabilidad a los fenómenos climáticos extremos que los países deben entender como una advertencia para estar preparados para eventos climáticos más frecuentes y/o más severos en el futuro. (Germanwatch, 2019). en los países pobres y en vías de desarrollo por el componente de vulnerabilidad ${ }^{13}$ climática que poseen ante estos eventos.

Ver tabla 1 y mapa del mundo con el Índice de Riesgo Climático Global para los años 1998-2017.

El impacto en la región centroamericana ha sido visible y palpable como en otros países y regiones por el aumento en un primer momento de las temperaturas, la cual ha sufrido un incremento de entre $0.7{ }^{\circ} \mathrm{C}$ y $1{ }^{\circ} \mathrm{C}$ desde mediados de los años setenta y por una tendencia decreciente en las precipitaciones anuales; de igual manera, se ha presentado un impacto significativo en el recurso hídrico que ha tenido una disminución radical, ocasionada no sólo por la disminución de las precipitaciones, sino por la creciente demanda tanto para actividades económicas y de la población que va en aumento con el paso de los años (este punto es crítico, ya que sin considerar los impactos que podría traer consigo el cambio climático, la disponibilidad del agua se vuelven preocupante por el alto índice de pobreza de la región y por los impactos acumulados de fenómenos extremos que puedan presentarse), razón por la cual su gestión eficiente es parte de la Agenda 2030 al ser incluida como parte de los ODS. 
Tabla 1

Ranking Índice de Riesgo Climático Global, 2017

\begin{tabular}{c|c|c|c|c|c}
\hline Ranking & País & Valor IRC & $\begin{array}{c}\text { Muertos } \\
\text { (promedio anual) }\end{array}$ & $\begin{array}{c}\text { Muertos por 100 } \\
\text { 000 habitantes } \\
\text { (promedio anual) }\end{array}$ & $\begin{array}{c}\text { Pérdidas en } \\
\text { millones de } \\
\text { dólares (PPA) } \\
\text { (promedio anual) }\end{array}$ \\
\hline 2 & Honduras & 13.00 & 302.450 & 4.215 & 556.56 \\
\hline 6 & Nicaragua & 20.00 & 163.600 & 2.945 & 223.25 \\
\hline 14 & Guatemala & 36.50 & 98.600 & 0.709 & 394.455 \\
\hline 16 & El Salvador & 37.67 & 32.300 & 0.530 & 277.55 \\
\hline 30 & Belice & 47.67 & 2.350 & 0.759 & 66.154 \\
\hline 92 & Costa Rica & 87.67 & 6.700 & 0.153 & 66.303 \\
\hline 98 & Panamá & 91.17 & 10.000 & 0.285 & 38.097 \\
\hline
\end{tabular}

Fuente: Elaboración propia de autor con datos obtenidos de informe “Global Climate Risk Index 2019” de Germanwatch.

\section{e) Mapa del mundo con el Índice de Riesgo Climático Global para los años 1998-2017.}

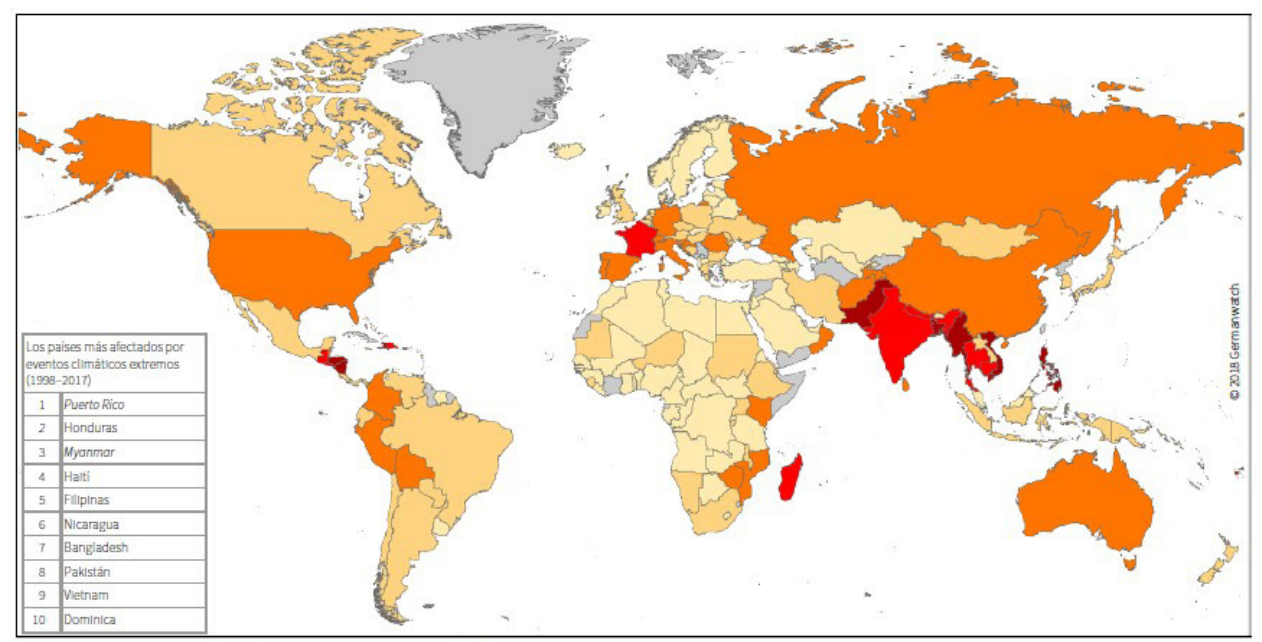

En cursiva: Las paises en que $90 \%$ de las pérdidas / los casos martales ocurrieron dentro de un año/ o un evento.

Indice de Riesgo Climático Global: Ranking 1998-2017

1.10 $\square 11-20 \square 21-50 \square 51-100 \square>100 \square$ Datos no disponinibles

Figura 5. d) Mapa del mundo con el Indice de Riesgo Climático Global entre los años de 1998 y 2017, se indica en letra cursiva los países en que el $90 \%$ de las pérdidas / los casos mortales ocurrieron dentro de un año o un evento, mostrando evidencia en términos relativos, que los países más pobres son golpeados más severamente. Fuentes: Germanwatch y Munich Re NatCatSERVICE*.

* Nota: Una de las principales bases de datos a nivel mundial dedicada al análisis y evaluación de desastres naturales. 
Tabla 2

Centroamérica: Recursos de agua por pais

\begin{tabular}{c|c|c|c|c|c|c}
\hline País & $\begin{array}{c}\text { Total recursos } \\
\text { hídricos } \\
\text { internos } \\
\text { IRWR* 10^9 } \\
\mathbf{m 3} \text { año }\end{array}$ & $\begin{array}{c}\text { Total de } \\
\text { recursos } \\
\text { externos } \\
\text { renovables } \\
\mathbf{1 0}^{\wedge 9} \mathbf{m} \text { año }\end{array}$ & $\begin{array}{c}\text { Total de } \\
\text { recursos } \\
\text { hídricos 10^9 } \\
\mathbf{m 3} \text { año }\end{array}$ & $\begin{array}{c}\text { Tasa } \\
\text { dependencia } \\
\text { \% }\end{array}$ & $\begin{array}{c}\text { Extracción } \\
\text { anual de agua } \\
\text { dulce }\end{array}$ & $\begin{array}{c}\text { Total de } \\
\text { recursos de } \\
\text { agua per } \\
\text { cápita m3/ } \\
\text { hab/año }\end{array}$ \\
\hline Belice & 15.25 & 6.47 & 21.72 & 29.790 & 0.8 & 65,452 \\
\hline Costa Rica & 113.00 & 0.00 & 113.00 & 0 & 2.4 & 23,194 \\
\hline El Salvador & 15.63 & 10.64 & 26.27 & 40.50 & 3.8 & 4,144 \\
\hline Guatemala & 109.20 & 18.71 & 127.91 & 14.63 & 2.6 & 8,269 \\
\hline Honduras & 90.66 & 1.50 & 92.16 & 1.63 & 1.2 & 11,381 \\
\hline Nicaragua & 156.20 & 8.31 & 164.51 & 5.05 & 0.7 & 27,056 \\
\hline Panamá & 136.60 & 2.70 & 139.30 & 1.94 & 0.3 & 36,051 \\
\hline
\end{tabular}

Fuente: Elaboración propia de autor con datos obtenidos de AQUASAT** y de Global Water Parnetrship (GWP) de Centroamérica (2017).

*Nota: Según la FAO (Organización de las Naciones Unidas para la Alimentación y la Agricultura), el término de Recursos Hídricos Internos Renovables (Internal Renewable Water Resources) es el flujo anual medio de los ríos y de las recargas de acuíferos generados por la precipitación endógena.

** Nota: Sistema mundial de información de la FAO sobre recursos hídricos y gestión agrícola del agua.

La vulnerabilidad climática también es visible en los ecosistemas marinos y costeros de la región, los cuales se han visto mermados por la presencia humana, a través de diferentes actividades tales como turismo, expansión urbana no planificada, extracción de peces para satisfacer la demanda creciente de productos marinos, entre otras actividades socioeconómicas. Uno de los sectores que sin lugar ha sufrido mayores consecuencias es la agricultura, en gran medida porque éstas dependen directamente del clima propio de la región; es lógico deducir en ese sentido, que las variaciones en el clima (lluvias intensas, períodos de sequías, cambios en el patrón de las lluvias, etc.) que se presenten incidirán directamente en la capacidad de producción de la región y del sector como tal; evidencia de ello es que se han presentado cambios drásticos en el clima en años recientes que han afectado enormemente la producción, ocasionándole pérdidas y daños en algunos casos irreparables. Otro de los sectores que se ve afectado por estos fenómenos es el energético, debido a que la disminución en la afluencia de los ríos ha traído consigo la reducción en la generación de la energía eléctrica en muchas de sus represas principales como la de Cerrón Grande en El Salvador y Chixoy en Guatemala (CEPAL, 2015b). Por último, los ecosistemas propios de la región, incluidos sus bosques tropicales y que tienen según estimaciones el $7 \%$ de la biodiversidad del planeta, se han visto mermados por la tala indiscriminada, la expansión de las tierras destinadas para uso agrícola y el uso intensivo de pesticidas y fertilizantes; lo que ha pronosticado un impacto significativo entre 2005 y 2100, 
período en el cual la región perdería la tercera parte de sus bosques tropicales, un 13\% de la biodiversidad y el $80 \%$ de sus pastizales, sabanas y arbustales, creciendo en el mismo período la tierra destinada para fines agrícolas en un 50\% (CEPAL, 2012a).

\section{El desarrollo y su relación con el cambio climático en la región}

El crecimiento económico experimentando por la región en los últimos años se ha mantenido levemente, gracias a la lenta recuperación de las principales economías mundiales y de las economías emergentes y en desarrollo; a grandes rasgos la economía a nivel mundial según estudios de la CEPAL (2019) registró una crecimiento en 2018 del 3.2\% en contraste con el crecimiento reportado en 2017 del 3.1\%; en ese sentido el crecimiento para la región de América Latina y el Caribe tuvo una expansión frágil del 1.2\% en 2018, que refleja una leve desaceleración con respecto al período anterior donde fue del 1.3\%; en la misma sintonía, la región de Centroamérica como tal, tuvo un crecimiento del $3.2 \%$ en contraste con el 3.4\% del 2017. En este mismo estudio, cabe destacar el crecimientos estimado para el pasado 2019, donde se preveía que la región de América Latina y el Caribe como tal tendría un repunte del 1.7\%; mientras que Centroamérica y la República Dominicana tendrían un repunte del 4\%; vale mencionar, que en una posterior revisión de la CEPAL (11 de abril de 2019) se actualizaron las proyecciones y la estimación para la región de América Latina y el Caribe tuvo una leve contracción del $1.7 \%$ al 1.3\%, ocasionadas por el complejo escenario externo y las dinámicas domésticas (gasto e inversión) que se han venido presentando en algunos países; en esta misma actualización, las estimaciones para Centroamérica para 2019 bajaron a un 3.1\%, prácticamente por bajas en el crecimiento que tendrán sus países ocasionadas por la leve desaceleración de uno de sus principales socios comerciales como lo es Estados Unidos, tanto en el comercio como en otro de sus principales componentes que es el flujo de efectivo proveniente de las remesas familiares y que conforman gran parte de sus ingresos anuales y del Producto Interno Bruto (PIB) de sus países, y que según estimaciones de CEPAL alcanzaron un 11\% en 2018. Ver Gráfico 2.

Los números anteriores reflejan el crecimiento económico que ha tenido la región centroamericana, los cuales fueron hasta cierto punto satisfactorios considerando el entorno actual, y gracias principalmente al apoyo del comercio intrarregional, al flujo de exportaciones e importaciones y a las remesas familiares, ya que ha permitido que se mejoren muchas condiciones dentro de su población y en cada uno de los países que la conforman; sin embargo, no se puede dejar de lado que todo ese escenario impacta en otras áreas, como en lo referente a la contaminación en general proveniente de las actividades comerciales internas, entre sus países o con el exterior, y por ende, con el cambio climático. Para validar este punto es conveniente analizar y darnos cuenta que el incremento de la población demanda consigo recursos para la satisfacción de todas sus necesidades básicas, aparte que gran parte de ellas estarán asentadas en las áreas urbanas de las economías en desarrollo -se estima que habrá 1700 millones de personas más para el año 2040- lo cual elevará en más de un cuarto la demanda energética mundial; el tema con esto, y si se trae al plano las energías renovables -un elemento clave para la adaptación al cambio 


\section{Gráfico 2}

Crecimiento económico de los países de Centroamérica y de la región de América Latina y el Caribe, 2010-2018

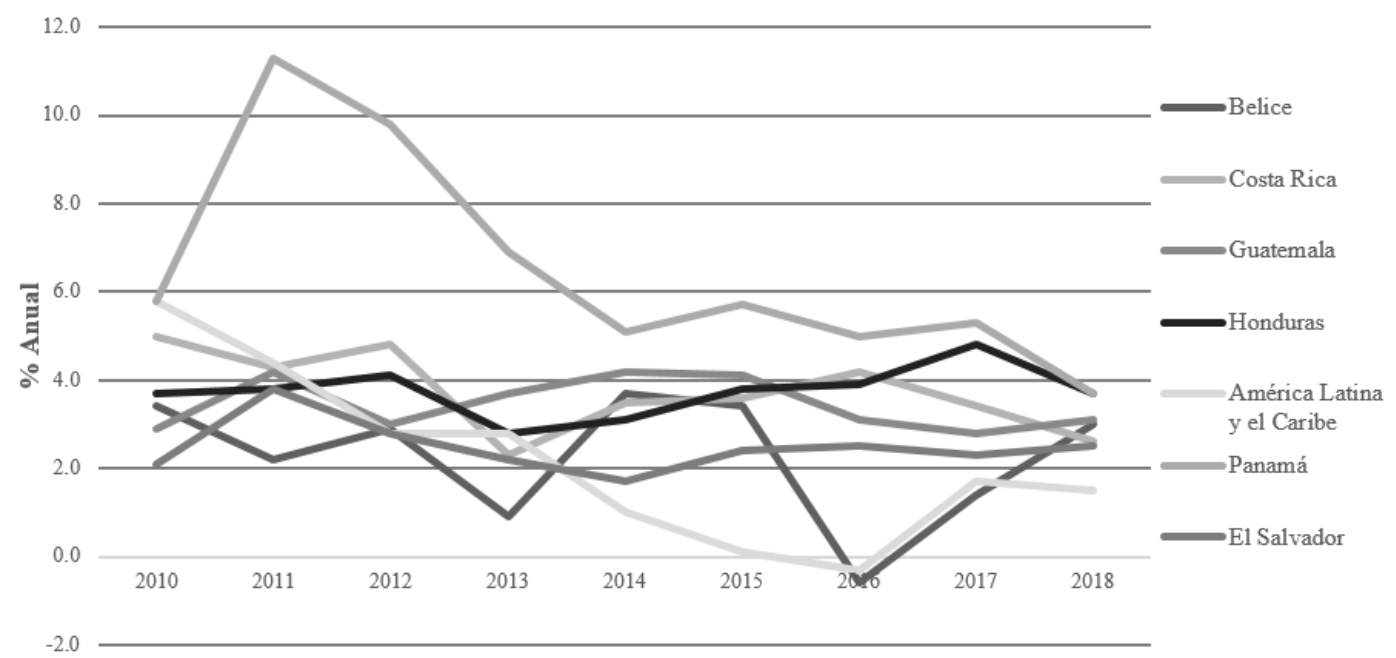

Fuente: Elaboración propia del autor con datos obtenidos del Banco Mundial de la herramienta DataBank y de la sección de "Indicadores del desarrollo mundial".

climático según las Naciones Unidas- es que en estimaciones realizadas, hasta el momento el uso de combustibles fósiles ha crecido más y consecuentemente, y en términos porcentuales la participación de las energías renovables es mucho menor; claro ejemplo es el uso del carbón como fuente de energía, que en 2017 y después de dos años a la baja, tuvo un repunte nuevamente; sin embargo, en este nuevo escenario que se está forjando, la marea ascendente de electricidad, energías renovables y mejora en eficiencia ha contenido el crecimiento del consumo de carbón, claro ésta que se necesita un impulso mucho mayor de éstos en el escenario mundial (Agencia Internacional de Energía (IEA por sus siglas en inglés), 2018). Vale aclarar, que aún en un escenario de desarrollo sostenible, el gas natural, el carbón y el petróleo seguirán respondiendo hasta el 2040 a una parte fundamental de la demanda energética mundial; producto de la creciente demanda que se avecina o de proyectos en curso relacionados a este tipo de energías, ejemplo, la creciente creación de una serie de plantas centrales de carbón que tienen un promedio de vida de 15 años en Asia y de 40 años en las economías avanzadas; la presunción y "promesa” es que después de dicho período su consumo empezará a disminuir. Ver Gráfico 3.

Es claro que el entorno actual en que se vive es insostenible y a pesar de que pueda haber períodos de bonanza en otros sectores, se está afectando drásticamente los recursos naturales con que se cuenta; por lo que el estilo es insostenible y se erosiona la débil sostenibilidad con la que se cuenta actualmente en la región. Ver Figura 6. 


\section{Gráfico 3}

Consumo de energía eléctrica por fuente de origen en América Latina, 1990-2016

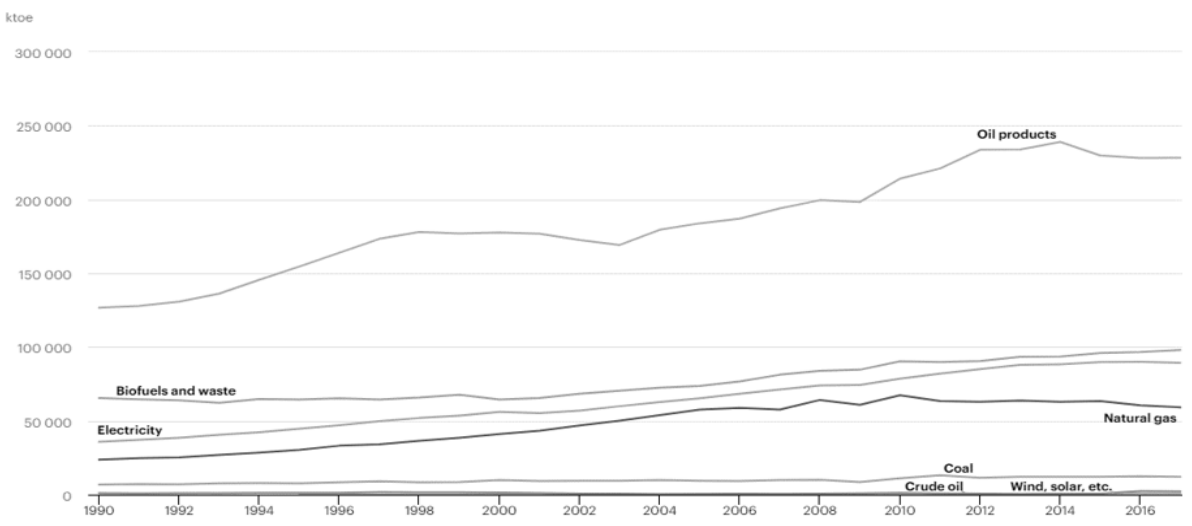

Fuente: Elaboración propia del autor con datos obtenidos de IEA de la sección de "Datos y estadísticas" y registrada en la unidad de energía ktoe (Kilotonelada equivalente de petróleo).

\section{f) Impactos generalizados atribuidos al cambio climático por regiones.}

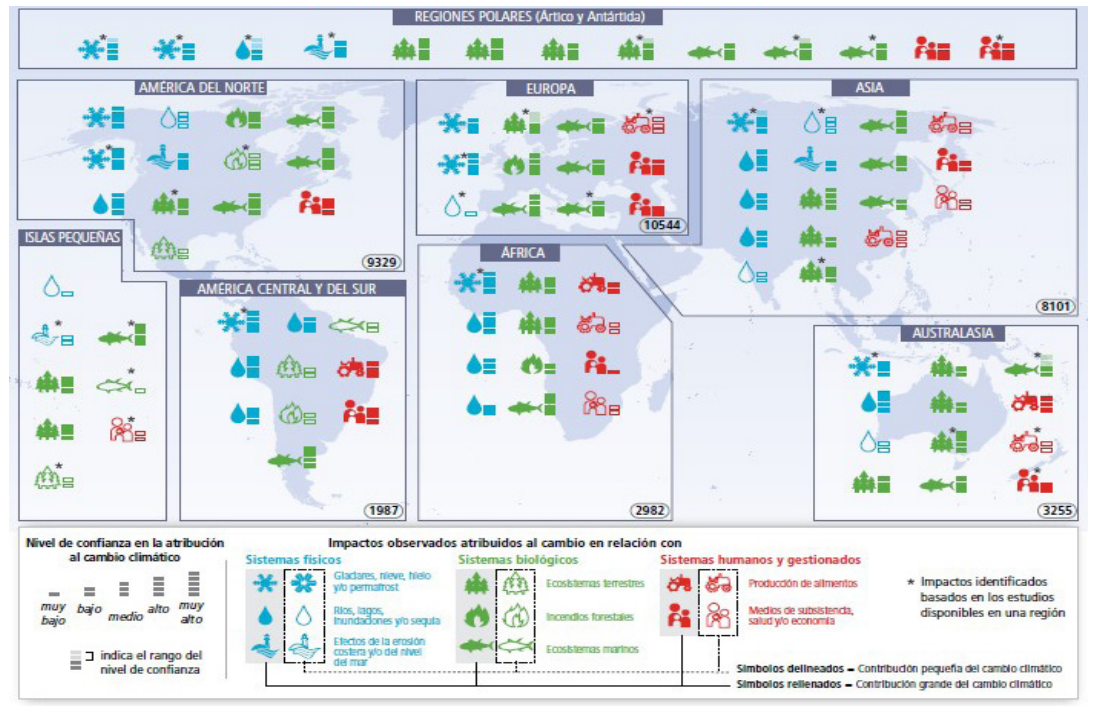

Figura 6. Sobre la base de la documentación científica del cuarto informe de evaluación del IPCC se desglosan un mayor número de impactos atribuibles al cambio climático en las distintas regiones, los que se detallan en el quinto informe del IPCC (2014) que ha servido de referencia. Se aclara que estas cifras proporcionan una idea general de la documentación científica disponible sobre el cambio climático en las distintas regiones. 


\section{De la planificación a la acción: No hay más tiempo que perder}

Los esfuerzos para luchar contra el cambio climático datan de hace muchas décadas; el primer intento se dio en el año de 1949 en la Conferencia Científica de las Naciones Unidas sobre Conservación y Utilización de los Recursos ${ }^{14}$; sin embargo, su enfoque estaba dirigido fundamentalmente en cómo gestionarlos correctamente en beneficio del desarrollo económico y social, pero sin preocuparse por su conservación (Jackson, s.f.); no es hasta el año de 1972 cuando se encuentra el primer fruto tangible en el momento en que se celebra la Conferencia de las Naciones Unidas sobre el Medio Humano (CNUMH) ${ }^{15}$, de la cual emanó la "Declaración de Estocolmo" conformada por 26 principios y donde se reconocía el derecho a conservar un ambiente natural saludable y se hacía mención que los cambios provocados por el hombre en el medio ambiente habían ocasionado problemas urgentes en los distintos países, problemas que sólo podían ser resueltos mediante la cooperación internacional.

Este primer gran paso dado por las Naciones Unidas dio lugar a que en ese mismo año, la Asamblea General de las Naciones Unidas creara por recomendaciones brindadas en la

14 Celebrada en_Nueva York (Estados Unidos) del 17 de agosto al 6 de septiembre de 1949.

15 Realizada en Estocolmo (Suecia) del 5 al 16 de junio de 1972, contó con la participación de 113 Estados miembros de las Naciones Unidas, así como miembros de organismos especializados de la Organización y se considera como la primera gran conferencia de las Naciones Unidas sobre cuestiones ambientales internacionales (Naciones Unidas, s.f.).
CNMUH, el Fondo para el Medio Ambiente ${ }^{16}$ de las Naciones Unidas y el PNUMA que se financiaría con recursos de dicho Fondo y que se consolidaría como la autoridad ambiental líder en el mundo en esta temática.

Desde entonces, se han gestado una serie de acciones concretas y dirigidas en la lucha contra el cambio climático que lograron la creación del IPCC en 1988, y que permitieron que 1989 fuera un año decisivo para la temática del cambio climático por diversos sucesos que se suscitaron en aquel entonces y que permitieron que en 1992 se celebrara la Conferencia de las Naciones Unidas sobre el Ambiente y el Desarrollo, donde se establecieron una serie de marcos siendo uno de ellos la Convención Marco de las Naciones Unidas sobre el Cambio Climático $(\mathrm{CMNUCC})^{17}$ para la adaptación, pérdidas $\mathrm{y}$ daños y que contiene lineamientos claros sobre la estabilización de las concentraciones atmosféricas de GEI a un nivel que no pusiera en riesgo el sistema climático.

Las bases que se sentaron dieron lugar a la celebración periódica de diferentes cumbres climáticas ${ }^{18}$ con la finalidad de llegar a acuerdos entre los Estados miembros en distintos rubros relacionados a esta lucha; es así como en 2015 se concreta la Agenda 2030 para el Desarrollo

16 Es la mayor fuente de financiación flexible del PNUMA y el principal apoyo para la labor que se desempeña a nivel mundial, cuenta con el respaldo de 193 Estados miembros (Programa para el Medio Ambiente, s.f.).

17 Celebrada en Río de Janeiro (Brasil) del 3 al 14 de junio de 1992, reafirmó el CNUMH.

18 Conferencias internacionales que no son más que reuniones organizadas por las Naciones Unidas en este tema. 
Sostenible y se logra el Acuerdo de París ${ }^{19}$ que entre sus líneas principales trata de mantener el aumento de la temperatura media mundial muy por debajo de $2{ }^{\circ} \mathrm{C}$ con respecto a los niveles preindustriales, $\mathrm{y}$ de limitar ese aumento a una temperatura promedio de $1.5{ }^{\circ} \mathrm{C}$; esto, considerando que de exceder los límites definidos no tendríamos capacidad de repuesta para afrontar las terribles consecuencias que pudiesen desatarse; se le reconoce como el primer acuerdo vinculante sobre el clima. Vale mencionar que en años recientes se sufrió un duro revés en relación con el Acuerdo de París con la salida de Estados Unidos en $2017^{20}$; desde entonces, las acciones a nivel mundial han seguido su curso y no se ha quitado el dedo del renglón al seguir buscando los compromisos entre los países.
19 En la Conferencia de París sobre el Clima (Reconocida como COP21) celebrada en diciembre de 2015, 195 países firmaron el primer acuerdo vinculante mundial sobre el clima; el mismo, establece un puente entre las políticas actuales y la neutralidad climática que debe existir a finales de este siglo (Comisión Europea, s.f.).
20 Estados Unidos con la entrada del Gobierno de Donald Trump, decidió salirse del Acuerdo de París el 1 de junio de 2017 al considerarla como una injusta carga económica impuesta a sus empresas y contribuyentes.

\section{Tabla 3}

Eventos importantes relacionados a la lucha contra el cambio climático a nivel mundial desde 1972

\begin{tabular}{|c|c|c|}
\hline Año & Evento & Comentarios \\
\hline 1972 & $\begin{array}{l}\text { Conferencia de las Naciones Unidas sobre el } \\
\text { Medio Humano. }\end{array}$ & Se crean las bases para la lucha contra el cambio climático. \\
\hline 1979 & $\begin{array}{l}\text { Primer Conferencia Mundial sobre el Clima en } \\
\text { Ginebra. }\end{array}$ & $\begin{array}{l}\text { Se considera al cambio climático como una amenaza real } \\
\text { para el planeta. }\end{array}$ \\
\hline 1988 & Creación del IPCC. & $\begin{array}{l}\text { Se conforma de expertos que darán seguimiento } \\
\text { constante a la crisis. }\end{array}$ \\
\hline 1989 & Resolución 44/207 & $\begin{array}{l}\text { Se prueba la solicitud del Consejo de Administración del } \\
\text { PNUMA para iniciar junto con la OMM los preparativos } \\
\text { para el CMNUCC. } \\
\text { Declaración de Male }{ }^{1} \text {. } \\
\text { Declaración de Helsinki }{ }^{2} \text {. } \\
\text { Protocolo de Montreal }\end{array}$ \\
\hline 1992 & $\begin{array}{l}\text { Conferencia de las Naciones Unidas sobre el } \\
\text { Medio Ambiente y el Desarrollo. }\end{array}$ & $\begin{array}{l}\text { Se adopta el plan conocido como Agenda } 21^{4} \text {. } \\
\text { CMNUCC. } \\
\text { Convenio de las Naciones Unidas sobre la Diversidad } \\
\text { Biológica (CNUDB) }{ }^{5} \text {. } \\
\text { Convención de las Naciones Unidas de Lucha contra la } \\
\text { Desertificación (CNULD) }{ }^{6} \text {. }\end{array}$ \\
\hline 1995 & Primer Conferencia de las Partes (COP) en Berlín. & $\begin{array}{l}\text { Se celebra la primera conferencia de las partes de la } \\
\text { CMNUCC. } \\
\text { Mandato de Berlín }{ }^{7} \text {. }\end{array}$ \\
\hline 1997 & Protocolo de Kyoto ${ }^{8}$ (COP3). & $\begin{array}{l}\text { Los países industrializados adquieren un compromiso } \\
\text { concreto y un plan de acción. }\end{array}$ \\
\hline
\end{tabular}




\begin{tabular}{|c|c|c|}
\hline Año & Evento & Comentarios \\
\hline 2001 & Cumbre de Marrakech (COP7). & Fondo de Adaptación?. \\
\hline 2002 & Cumbre de Johannesburgo (COP8). & Se renueva el compromiso político con el desarrollo sostenible \\
\hline 2007 & Cumbre de Bali (COP13). & $\begin{array}{l}\text { Se inicia proceso de negociación para el segundo período } \\
\text { de cumplimiento del Protocolo de Kyoto. }\end{array}$ \\
\hline 2009 & Cumbre de Copenhague (COP15). & $\begin{array}{l}\text { Firma del acuerdo de Copenhague que fija el límite } \\
\text { máximo de incremento permitido de la temperatura } \\
\text { media global en } 2^{\circ} \mathrm{C} \text {. }\end{array}$ \\
\hline 2010 & Cumbre de Cancún (COP16). & Fondo Verde para el Clima $(\mathrm{FVC})^{10}$. \\
\hline 2011 & Cumbre de Durban (COP17). & $\begin{array}{l}\text { Se aprueba una hoja de ruta }{ }^{11} \text { que obliga a comprometerse } \\
\text { a los principales contaminadores: China, Estados Unidos } \\
\text { e India. }\end{array}$ \\
\hline 2013 & Cumbre de Varsovia (COP19). & $\begin{array}{l}\text { Se buscan acuerdos en relación a la reducción de } \\
\text { emisiones; pero muchos miembros se oponen a ellos. }\end{array}$ \\
\hline 2015 & $\begin{array}{l}\text { Cumbre de Desarrollo Sostenible } \\
\text { XXI Conferencia Internacional sobre Cambio } \\
\text { Climático (COP21). }\end{array}$ & $\begin{array}{l}\text { Agenda } 2030 \text { para el Desarrollo Sostenible. } \\
\text { Acuerdo de París. }\end{array}$ \\
\hline 2016 & Cumbre de Marrakech (COP22). & Se celebra la entrada en vigor del Acuerdo de París. \\
\hline 2017 & Cumbre de Boon (COP24). & $\begin{array}{l}\text { Plan de Acción de Género }{ }^{12} \text {. } \\
\text { Alianza Global contra el Carbón }{ }^{13} \text {. }\end{array}$ \\
\hline 2019 & Cumbre de Madrid (COP25). & Acuerdo "Chile-Madrid. Tiempo de Actuar ${ }^{14 "}$. \\
\hline
\end{tabular}

Fuente: Elaboración propia del autor con datos obtenidos de la biblioteca digital de las Naciones Unidas y de informe relacionado a las cumbres llevadas a cabo por las Naciones Unidas (Vengoechea, 2012).

\section{Notas de tabla}

1 Documento que trata sobre el calentamiento de la atmósfera en todo el mundo y el aumento del nivel del mar.

2 Documento que trata sobre la protección de la capa de ozono.

3 Documento que trata sobre las sustancias que agotan la capa de ozono, nació en 1987 y entró en vigor en 1989.

4 Plan de acción propuesto por las Naciones Unidas que buscaba un desarrollo más sostenible para el siglo XXI, fue aprobado y firmado por 173 miembros.

5 Tratado internacional para conservar la diversidad biológica; está compuesto por el Protocolo de Cartagena sobre Seguridad de la Biotecnología y el Protocolo de Nagoya sobre acceso a los recursos genéticos.

6 Acuerdo que entró en vigencia en 1996 para promover una respuesta global ante la desertificación. 
7 En la primera conferencia se adopta este mandato que exige a las partes que inicien negociaciones para reducir emisiones más allá del 2000 mediante objetivos cuantitativos y plazos concretos.

8 Acuerdo creado para reducir las emisiones de GEI que causan el calentamiento global, aprobado en 1997 y con entrada en vigencia hasta 2005.

9Tiene como objetivo la financiación de proyectos y programas emprendidos por países en desarrollo.

10 Fondo creado con el mandato de promover un cambio de paradigma hacia la reducción de las emisiones y brindar apoyo a los países en desarrollo para la adaptación al cambio climático.

11 Propuesta por la Unión Europea que buscaba un nuevo acuerdo global vinculante de reducción de GEI.

12 Plan que busca integrar a las mujeres en la toma de decisiones relacionadas al cambio climático.

13 Powering Past Coal Alliance, alianza de países que busca la eliminación rápida y gradual del carbón como fuente de energía.

14 Acuerdo que plantea entre algunas cuestiones el compromiso para 2020 para que los países presentan planes de reducción de GEI más ambiciosos para responder de una mejor manera a la emergencia climática; sin embargo, no se contó con el respaldo de Estados Unidos, China e India en relación a cumplir este compromiso.

Sobre el cambio climático dentro de la región centroamericana, se han llevado a cabo muchas acciones para tratar de mermar su impacto; algunas datan desde 1993 cuando los países de la región firmaron el Convenio Regional sobre Cambios Climáticos, mismo que fue ratificado en 2008 y donde se manifiesta explícitamente la gravedad del problema y el compromiso por enfrentarlo de la mejor forma posible y que al final dio paso al desarrollo de la Estrategia Regional de Cambio Climático (ERCC) liderado por el Comité Técnico Regional de Cambio Climático; la preparación de dicha estrategia fue catalogada como el resultado de un proceso intenso de consultas y aportes de los países miembros del Sistema de la Integración Centroamericana (SICA) que se entregó en 2010 y que fue aprobado en 2011.

Recientemente, esta estrategia ha sufrido cambios para armonizar los mismos con el Acuerdo de París, con los ODS de las Naciones Unidas y con las acciones que se están gestando a nivel regional en esa misma línea, contando con la particularidad del apoyo recibido de parte de la Unión Europea (SICA, 11 de mayo de 2018). En el año 2019 se presentó la nueva versión de la ERCC, que se formuló bajo el marco de transparencia y rendición de cuentas 
y que para su coordinación y conducción estratégica se propuso la conformación de un "Grupo de Trabajo del SICA para el Cambio Climático”, que estará conformado por la SG-SICA como coordinador del Grupo, la Comisión Centroamericana de Ambiente y Desarrollo (CCAD) ${ }^{21}$, en calidad de Secretario Técnico y coordinador del subsistema ambiental; y la Secretaría de

21 Nació con la misión de desarrollar un régimen regional de cooperación e integración ambiental que contribuya a mejorar la calidad de vida de las poblaciones de sus Estados miembros.
Integración Económica Centroamericana $(\text { SIECA })^{22}$ y la Secretaría de Integración Social Centroamericana (SISCA) ${ }^{23}$ como coordinadores del subsistema económico y social respectivamente (SICA, 2019).

22 Órgano técnico y administrativo del Proceso de Integración
Económica Centroamericana que vela principalmente por el
cumplimiento de los instrumentos jurídicos de la integración cumplimiento de los
económica regional.

23 Nace en 1995 bajo el Protocolo de Tegucigalpa con el que se busca posicionar a lo social como un campo de trabajo central de los esfuerzos integracionistas.

\section{Tabla 4}

Eventos importantes relacionados a la lucha contra el cambio climático en Centroamérica

\begin{tabular}{|c|c|c|}
\hline Año & Logros & Comentarios \\
\hline 1989 & Constitución de la CCAD. & $\begin{array}{l}\text { Surge por el interés en el desarrollo sostenible y preocupación } \\
\text { por el cambio climático. }\end{array}$ \\
\hline 1993 & Convenio Regional sobre Cambios Climáticos. & $\begin{array}{l}\text { Nace por la intención de los Estados de proteger el sistema } \\
\text { climático en beneficio de las generaciones presentes y futuras. }\end{array}$ \\
\hline 1994 & Alianza para el Desarrollo Sostenible. & $\begin{array}{l}\text { Acuerdo que tiene la finalidad de inducir un proceso de } \\
\text { cambio progresivo en la calidad de vida del ser humano. }\end{array}$ \\
\hline 1999 & $\begin{array}{l}\text { Plan Ambiental de la Región Centroamericana } \\
\text { (PARCA) 2000-2004. }\end{array}$ & $\begin{array}{l}\text { Constituyó un ejercicio de creación capacidades para abordar } \\
\text { los retos ambientales de la región y buscaba operativizar la } \\
\text { ALIDES e iniciar la consolidación del CCAD. }\end{array}$ \\
\hline 2004 & PARCA 2004-2009. & $\begin{array}{l}\text { Se enfocó en la formulación y validación de instrumentos de } \\
\text { política regionalmente armonizados }\end{array}$ \\
\hline 2008 & Declaración de San Pedro Sula. & Se aprueban lineamientos para la creación de la ERCC. \\
\hline 2010 & $\begin{array}{l}\text { Estrategia Regional de Cambio Climático. } \\
\text { PARCA 2010-2014. }\end{array}$ & $\begin{array}{l}\text { Instrumento orientador de las medidas y acciones regionales } \\
\text { complementarias en el tema medioambiental. } \\
\text { Se enfocó en la gobernanza ambiental con un modelo de } \\
\text { gestión basado en la aplicación y cumplimiento ambiental. }\end{array}$ \\
\hline 2015 & Estrategia Ambiental Regional Marco. & $\begin{array}{l}\text { Documento Marco que tenía la finalidad de establecer una } \\
\text { vinculación directa entre las estrategias regionales sectoriales. }\end{array}$ \\
\hline 2018 & $\begin{array}{ll}\text { Estrategia Regional de Cambio Climático } \\
\text { Actualizada 2018-2022 }\end{array}$ & Instrumento actualizado de ERCC. \\
\hline 2019 & $\begin{array}{l}\text { Estrategia Energética Sustentable Centroamericana } \\
2020 .\end{array}$ & $\begin{array}{l}\text { Informe desarrollado por SICA y CEPAL en relación a las } \\
\text { energías renovables. }\end{array}$ \\
\hline
\end{tabular}

Fuente: Elaboración propia del autor con datos obtenidos de informes del SICA y de CCAD (2009), se hace la aclaración que en el mismo se mencionan los principales eventos a criterio de autor; sin embargo, se han desarrollado medidas nacionales $\mathrm{y}$ regionales a su vez en la misma línea de acción. 
Por otra parte, para la región de América Latina y el Caribe, la Unión Europea preparó en su momento el Programa Indicativo Plurianual Regional para América Latina 2014-202024 que tiene objetivos sumamente ambiciosos relacionados con el cambio climático en la región, ya que tiene como finalidad contribuir al desarrollo de sociedad más resilientes y autosostenibles, a través de una mejor preparación de estas ante los fenómenos relacionados al cambio climático mediante la mitigación y la adaptación (Comisión Europea, 2014).

Como se observa, existe una buena proporción de medidas e iniciativas en la región centroamericana y en América Latina y el Caribe -así como ocurre en muchas otros regiones-; sin embargo, se presentan contradicciones entre las políticas de desarrollo y las políticas climáticas; en gran parte porque se considera desde una perspectiva netamente económica que la implementación efectiva de diversas acciones -que generalmente involucran los lineamientos brindados en el Acuerdo de París y la Agenda 2030 de las Naciones Unidastraerán consigo impactos directos en el PIB, el empleo, el comercio y la balanza de pagos; lo cual afectará a muchos sectores económicos que han crecido bajo la "vieja usanza" y sin considerar las implicaciones climáticas (Carlino, s.f.); es decir, sectores que han basado sus operaciones a través del uso intensivo de combustibles fósiles, de la extracción sin control de recursos naturales, de la deforestación, de la contaminación oceánica,

$24 \mathrm{El}$ programa proporciona directrices para el desarrollo de políticas de cooperación en la región con un presupuesto total de 925 millones de euros y cubre las siguientes áreas: Seguridad; buena gobernanza, responsabilidad e igualdad social; Crecimiento sostenible e integrador para el desarrollo humano; sostenibilidad medioambiental y cambio climático y educación superior (Fundación EU-LAC, 2017). etc.; en ese sentido, vale destacar lo indicado por el PNUMA en uno de sus últimos informes (2018) -en relación a la emisión de GEI-donde se establece que los compromisos actuales contenidos en las contribuciones determinadas a nivel nacional $(\mathrm{CDN})^{25}$ no son los adecuados para conseguir las metas en reducción o limitación del aumento de temperatura que se buscan para 2030, por lo que el PNUMA considera necesario aumentar las aspiraciones $y$ plantear medidas urgentes y sin precedentes por parte de las naciones.

Con base en lo anterior, surge un elemento sumamente importante para afrontar los retos asociados en la región centroamericana; el financiamiento climático ${ }^{26}$-ratificado en el Acuerdo de París- donde se establece que los flujos deben de ser consistentes y deben ir más allá de los esfuerzos previos; es decir, adicional a acuerdos establecidos anteriormente. En relación al último punto, es oportuno mencionar que aunque la región en general no es el principal destino del financiamiento climático, el mismo ha aumentado en los últimos años, con la aclaración de que, a pesar de su incremento, estos no son los suficientes para cubrir las necesidades de los países en esta área, principalmente por una serie de barreras que se han presentado a lo largo del tiempo a la hora de acceder a dichos fondos climáticos y que vale la pena mencionar: La primera de esas barreras

25 Las CDN constituyen el principal instrumento del Acuerdo de París, reflejan las acciones de cada país para enfrentar el cambio climático; es decir, los compromisos asumidos para la reducción de las emisiones de los GEI y la adaptación al cambio climático (Samaniego y otros, 2019).

26 La CMNUCC describe el financiamiento climático como el tipo de financiación local, nacional o transnacional que se usa para apoyar e implementar acciones de mitigación y adaptación al cambio climático, con recursos que provienen de fuentes públicas, privadas y alternativas (ParlAmericas, s.f.). 
es el acceso a financiamientos no reembolsables o donaciones, debido a que un problema particular de la mayoría de los países de la región de América Latina y el Caribe es que son considerados países de renta media ${ }^{27}$ o media-alta, lo que hace que los financiamientos que se puedan obtener estén dirigidos a estudios técnicos o a procesos de planificación, más no a su implementación; en segundo lugar, los países de la región enfrentan problemas relacionados a la escasez de recurso humano especializado, lo que permite que se presenten inconvenientes al momento de elaborar los proyectos por el hecho de que el personal debe de contar con conocimientos y/o experiencia en el sector; un tercer problema obedece a los trámites respectivos que deben de realizarse ante las organizaciones pertinentes como el FVC o el Fondo de Adaptación y que ha hecho que pocos países de la región puedan acceder a estos fondos (Scardamaglia, 2019). Estos problemas descritos pueden ser subsanados, siempre y cuando los países de la región presten especial atención a ellos y preparen mecanismos que permitan poder acceder a este tipo de financiamientos; sin lugar a dudas, el acceso a los mismos podría inclinar la balanza de forma positiva para la región y para sus habitantes.

\section{g) Fondos climáticos aprobados para Centroamérica por sector, 2003-2018.}

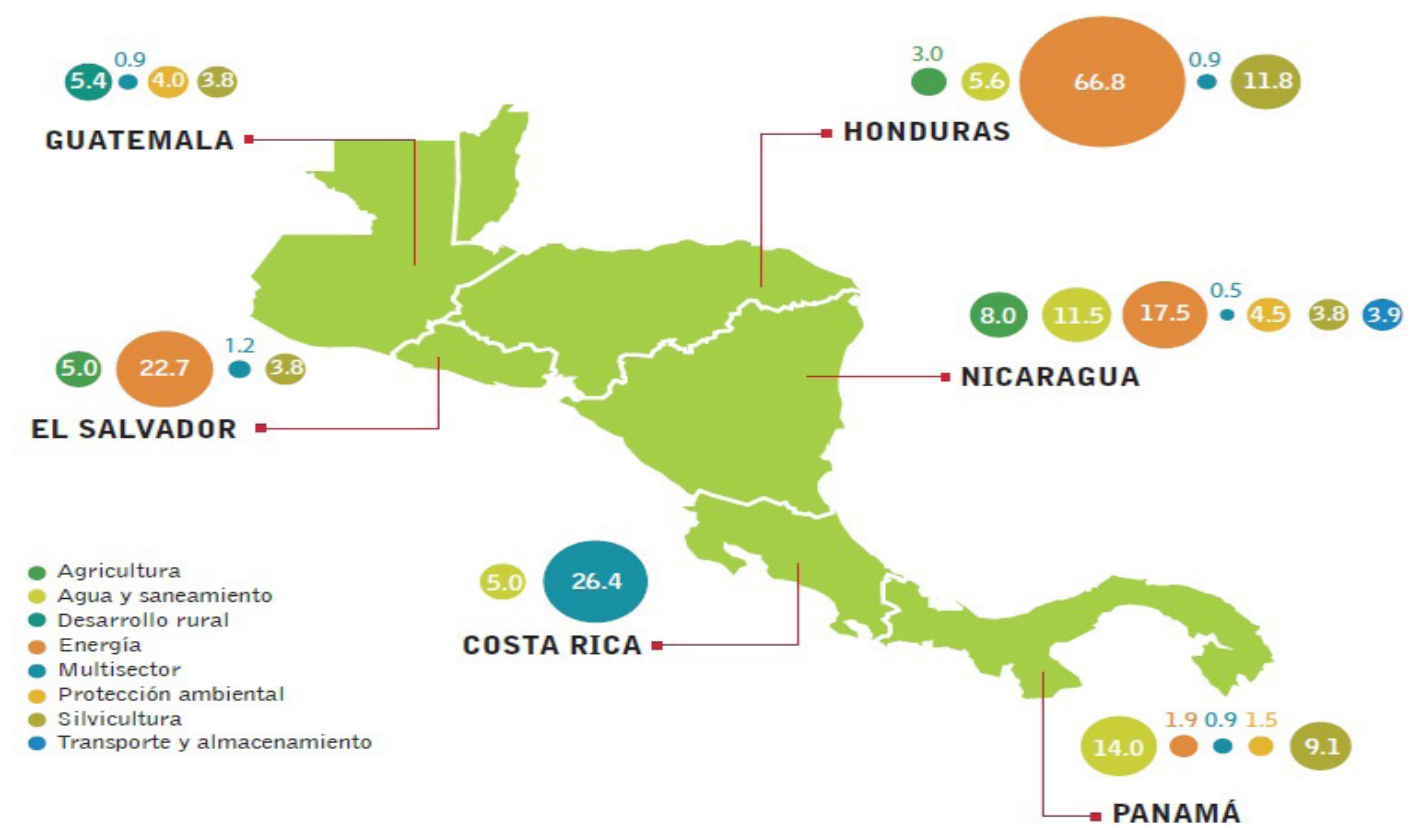

Figura 7. Fondos climáticos aprobados para Centroamérica en el período comprendido entre octubre 2003 y mayo de 2018 en millones de dólares y detallado por el sector destino. Se utiliza de fuente el informe de Heinrich Böll Stiftung Centroamérica (2019).

27 Se estima que más del 70\% de la población mundial vive en países de renta media; de ahí la necesidad de concentrar los recursos de cooperación internacional en el combate de la pobreza y en sus efectos inmediatos (CEPAL, 2012b). 


\section{Conclusiones}

Los resultados de este análisis permiten extraer conclusiones que se formulan seguidamente y que podrán ser contrastadas en estudios más complejos que contemplen más escenarios, componentes y una mayor representatividad de los datos:

1. Centroamérica es una de las regiones más expuestas al cambio climático a nivel mundial, lo que provoca que su muy diverso ecosistema sea vulnerable a los impactos provenientes de los fenómenos asociados que puedan presentarse, razón por la cual en los últimos años han presentado mayores manifestaciones tangibles como el aumento en la temperatura, aumento en el nivel del mar, aparición de más eventos extremos como sequías pronunciadas, en otros casos disminución en las precipitaciones anuales, atraso en los inicios de temporada lluviosa, entre otros eventos; en ese sentido, este complejo escenario afecta y lo hará cada vez en mayor proporción, a otros sectores relacionados; ya que permitirá que las vulnerabilidades que han sido identificadas adquieran mayor fuerza y afecten directamente las condiciones socioeconómicas de sus países y en especial de sus habitantes.

2. Si se considera que el desarrollo insostenible de la región centroamericana es un grave problema presente a nivel mundial (porque muchas regiones lo están haciendo de igual manera) es conveniente cambiar de una vez por todas el camino que se lleva; se tiene claro el daño que le hemos hecho al ecosistema y que debemos movernos a un desarrollo sostenible que permita que la región sea menos vulnerable a los efectos ocasionados por el cambio climático, manteniendo el crecimiento que se ha visto reflejado en otras áreas, y que permita que sus habitantes mantengan y mejoren, en la medida de lo posible, sus condiciones de vida.

3. A nivel mundial, el Acuerdo París plantea diversas acciones para tratar de lograr reducir el aumento en las temperaturas en los límites establecidos, plasmando diversos tipos de acciones que deben llevarse a cabo por todos los países; sin embargo, es necesario que exista mayor ambición y un compromiso real de parte de los países para tratar de reducir sus emisiones; especialmente de aquellos que han llegado a su límite máximo permitido; esto implica que se debe de fortalecer y ampliar las políticas nacionales encaminadas a dicho fin, incluyendo el desarrollo e implementación de otras políticas adicionales; y considerando el hecho que se ha planteado, que los límites de emisiones actuales definidos deben de reconsiderarse, debido al aumento de las emisiones registrados en los últimos años y que vislumbran un panorama nada alentador.

4. Por último, se identifican que existen muchos estudios, análisis y estrategias relacionadas a la lucha contra el cambio climático en la región centroamericana y en América Latina, que indican la gravedad del problema y la vulnerabilidad de la región ante los efectos relacionados; evidentemente, el no ejecutar las medidas correspondientes traerá costos excesivamente altos a la región y a sus habitantes; donde es clave quitarse el concepto de que este tema sólo le compete 
a organismos o instituciones ambientalistas, o incluso al Gobierno en sí, sino que es un tema crítico que atañe y compete a todos por igual; sin lugar a dudas esto traerá muchos desafíos para todos los sectores involucrados y para la población por lo que involucra, pero ya se cuenta con líneas directas de acción que no pueden esperar más tiempo para ser ejecutadas... ¡ $\mathrm{El}$ cambio es hoy!

\section{Recomendaciones}

La investigación permite brindar una serie de recomendaciones de parte del autor, las cuales podrían ser tomadas en cuenta en estudios posteriores, así como por los distintos actores inmersos en el estudio:

1. Se tiene claro la importancia del fenómeno en estudio y como este afecta a los países de la región centroamericana; de igual manera, se ha logrado visualizar una serie de medidas llevadas a cabo a nivel regional y global en la lucha contra el cambio climático; en ese sentido, se considera sumamente relevante que se continúe con la promoción de acciones políticas conjuntas y en la consolidación de propuestas regionales en ésta línea de acción entre los países de la región, las cuales deben ser llevadas a los distintos foros regionales e internacionales para solicitar el apoyo tanto de los otros Estados miembros como de los organismos y sus expertos en el análisis, diseño, implementación y control de proyectos de este tipo; y de ser posible, de su financiación.

2. Es necesario promover la investigación científica en la región; es decir, el desarrollo de estudios encaminados a evaluar el impacto del cambio climático en sus países y en los distintos ecosistemas que lo conforman, de esta manera se logrará contar con una base de datos sólida e histórica de conocimientos que podrá servir de base para futuros estudios y para el desarrollo de políticas y acciones climáticas concretas, las cuales podrían estar dirigidas a mitigación, adaptación y resiliencia de los fenómenos climáticos si es necesario; vale indicar que las acciones inmediatas pueden ir encaminadas a solventar problemas ya presentes; o en su defecto, a problemas previstos anteriormente.

3. Se está consciente que el cambio climático es un problema que no compete solamente a los Gobiernos o a los científicos; sino que involucra a todos por igual, ya que somos parte del problema y de la solución; por tal razón, se considera que tanto la educación como la concientización son componentes claves en este complejo escenario; por ende, es crítico continuar con la preparación de las generaciones futuras en esta línea de investigación; por lo que las políticas de acción deben ser claras y concretas en lo que se quiere lograr.

4. Debido a que gran parte de los países de la región tienen problemas para el acceso a los financiamientos climáticos, se considera clave que los países de la región centroamericana trabajen tanto en el fortalecimiento institucional para poder acceder a estos fondos, como en su ejecución eficiente. Una solución inmediata y viable podría ser trabajar de forma conjunta con distintos actores inmersos $y$ con instituciones o entidades ya acreditadas que transfieran dicho conocimiento $y / o$ experiencia en el rubro. 
5. Por último, como país y región se está consciente de los acuerdos que se han aprobado a nivel mundial, así como de los compromisos que se han adquirido en distintas áreas y líneas de acción; en ese sentido, se está consciente del reto que esto involucra tanto en el contexto político, económico, social, legal e institucional; por tal razón, se invita a cada uno de los países de la región y entidades pertinentes a la acción; es decir, a la generación de estrategias y mecanismos que permitan el logro de estos compromisos y al involucramiento sin distinción de todos los sectores de la sociedad en la consecución de los mismos.

\section{Bibliografía}

Carlino (s.f.). Análisis inicial de las implicaciones del Acuerdo de París en la mitigación y adaptación del cambio climático de América Latina y el Caribe. Ciudad de Panamá, Panamá: Programa de las Naciones Unidas. Recuperado de http://www. pnuma.org/cambio_climatico/publicaciones/ Acuerdo_de_Par\%C3\%ADs_-_Implicaciones_ en_ALC_-_Estudio_1.pdf

CCAD (2009). Plan Ambiental de la Integración Centroamericana. San Salvador, El Salvador: CCAD. Recuperado de https://www.sica.int/ download/?libr_56497_1_21012011.pdf

CCAD-SICA (2010). Estrategia Regional de Cambio Climático. Documento Ejecutivo. San Salvador, El Salvador. Recuperado de http:// bvssan.incap.int/local/cambio-climatico/ Estrategia-Regional-Cambio-Climatico.pdf
CEPAL (2012a). Cambio climático en Centroamérica. México D.F., México: Publicaciones de las Naciones Unidas. Recuperado de https://repositorio.cepal. org/bitstream/handle/11362/26122/1/ M20120031_es.pdf

CEPAL (2012b). Los paises de renta media: Un nuevo enfoque basado en brechas estructurales. Santiago de Chile, Chile: Publicaciones de las Naciones Unidas. Recuperado de https://repositorio.cepal. org/bitstream/handle/11362/13787/1/ S2012863_es.pdf

CEPAL (2015a). Cambio climático en Centroamérica, Impactos potenciales y opciones de política pública. México D.F., México: Publicaciones de las Naciones Unidas. Recuperado de http://repositorio.cepal. org/bitstream/handle/11362/39149/1/ S1501173_es.pdf

CEPAL (2015b). La economia del cambio climático en América Latina y el Caribe: paradojas $y$ desafíos del desarrollo sostenible. Santiago de Chile, Chile: Publicaciones de las Naciones Unidas. Recuperado de https://repositorio.cepal. org/bitstream/handle/11362/37310/S1420656_ es.pdf? sequence $=4 \&$ isAllowed $=y$

CEPAL (2018). La Agenda 2030 y los Objetivos de Desarrollo Sostenible. Una oportunidad para América Latina y el Caribe. Santiago de Chile, Chile: Publicaciones de las Naciones Unidas. Recuperado de https://repositorio. cepal.org/bitstream/handle/11362/40155/24/ S1801141_es.pdf 
CEPAL(2019).Balancepreliminar delaseconomías de Centroamérica y la República Dominicana en 2018 y perspectivas para 2019. México D.F., México: Publicaciones de las Naciones Unidas. Recuperado de https://repositorio.cepal.org/ bitstream/handle/11362/44485/1/S190116_ es.pdf

CEPAL (11 de abril de 2019). CEPAL rebaja sus estimaciones de crecimiento económico para América Latina y el Caribe: PIB aumentará 1,3\% en 2019. Recuperado de https://www.cepal.org/ es/comunicados/cepal-rebaja-sus-estimacionescrecimiento-economico-america-latina-caribepib-aumentara

CEPSA (2015). El Cambio Climático y los Gases de Efecto Invernadero (GEI) en Cepsa. Cepsa Dirección de Comunicación. Recuperado de https://www.cepsa.com/stfls/CepsaCom/ Coorp_Comp/Medio\%20Ambiente_ Seguridad_Calidad/Art\%C3\%ADculos/ Dossier-Cambio-Climatico-y-GEI.pdf

Colette, A. (2009) Estudios de caso: Cambio climático y Patrimonio Mundial. París, Francia: Centro del Patrimonio Mundial dela UNESCO (Organización de las Naciones Unidas para la Educación, la Ciencia y la Cultura). Recuperado de https://unesdoc. unesco.org/ark:/48223/pf0000183480

Comisión Europea (s.f.). Acuerdo de París. Recuperado de https://ec.europa.eu/clima/ policies/international/negotiations/paris_es

Comisión Europea (2014). Programa Indicativo Plurianual Regional para América Latina 20142020. Recuperado de https://eeas.europa.eu/ sites/eeas/files/mip_alr_vf_07_08_14_es_0.pdf
Evangelista,K.(2018).ESTADODELASNDC'S: Avances en la implementación de las contribuciones nacionalmente determinadas en materia de cambio climático en la región Centroamericana. San Salvador, El Salvador: Heinrich Böll Stiftung Oficinas Centroamérica. Recuperado de http:// sv.boell.org/sites/default/files/investigacion_ cambio_climatico_ca_220818.pdf

Fundación EU-LAC (2017). Vademécum sobre Programas de Cooperación de la UE con ALC. Hamburgo, Alemania. Recuperado de https://eulacfoundation.org/es/system/files/ vademecum_programas_cooperacion_ue_ lac.pdf

Fundación Heinrich Böll (2018). Atlas de los Océanos. Adenda Centroamérica 2018. San Salvador, El Salvador: Heinrich Böll Stiftung Oficinas Centroamérica. Recuperado de https://sv.boell.org/sites/default/files/atlas_ centroamerica_f.pdf

Germanwatch (2019a). Índice de Riesgo Climático Global 2019. Berlin, Alemania. Recuperado de https://www.germanwatch.org/ sites/germanwatch.org/files/Indice\%20de\%20 Riesgo\%20Climatico\%20Global\%202019\%20 -\%20Resumen_0.pdf

Germanwatch (2019b). Global Climate Risk Index 2019. Berlin, Alemania. Recuperado de https:/www.germanwatch.org/sites/ germ a nwatch.org/files / G 1 o b a $1 \% 20$ Climate\%20Risk\%20Index\%202019_2.pdf 
GWP Centroamérica (2017). La situación de los recursos hidricos en Centroamérica: Hacia una gestión integrada. Publicaciones de GWP Centroamérica. Recuperado de https://www. gwp.org/globalassets/global/gwp-cam_files/ situacion-de-los-recursos-hidricos_fin.pdf

Heinrich Böll Stiftung Centroamérica (2019). Financiamiento climático en Centroamérica: Una Mirada desde la descentralización energética. Recuperado de https://sv.boell.org/sites/default/ files/financiamiento_climatico_en_ca_-_una_ mirada_desde_la_descentralizacion_energetica.pdf

IEA (2018). World Energy Outlook. Publicaciones de la AIE. Recuperado de https://webstore.iea. org/download/summary/190?fileName=SpanishWEO-2018-ES.pdf

IPCC (2014). Cambio Climático 2014: Informe de sintesis. Contribución de los Grupos de trabajo I, II y III al Quinto Informe de Evaluación del Grupo Intergubernamental de Expertos sobre el Cambio Climático. [Equipo principal de redacción, R.K. Pachauri y L.A. Meyer (eds.)]. Ginebra, Suiza: IPCC. Recuperado de https://www.ipcc.ch/site/ assets/uploads/2018/02/SYR_AR5_FINAL_ full_es.pdf

IPCC (1 de mayo de 2015). Ficha informativa del IPCC: Fechas y momentos destacados de la historia del IPCC. Recuperado de https://www.ipcc.ch/ site/assets/uploads/2018/04/FS_timeline_es.pdf

Jackson (s.f.). De Estocolmo a Kyoto: Breve historia del cambio climático. Consultado en https:// www.un.org/es/chronicle/article/de-estocolmokyotobreve-historia-del-cambio-climatico
Naciones Unidas (s.f.). Declaración de Estocolmo. Biblioteca Audiovisual de las Naciones Unidas de Derecho Internacional. Recuperado de https:// legal.un.org/avl/pdf/ha/dunche/dunche_ph_s.pdf

OMM (2006). Vigilancia y alerta temprana de la sequí: Concepto, progresos y desafios futuros. Publicaciones de la Organización Meteorológica Mundial. Recuperado de http://www.wamis. org/agm/pubs/brochures/wmo1006es.pdf

OMM (2019). WMO GREENHOUSE GAS BULLETIN. Publicaciones de la Organización Meteorológica Mundial. Recuperado de https://library.wmo.int/index.php?lvl=notice_ display\&id=21620\#.Xrdx3GgzbIU

OMM (s.f.). Acerca de la OMM. Recuperado de https://public.wmo.int/es/acerca-de-la-omm

ParlAmericas (s.f.). Manual sobre financiamiento climático: Mecanismos y oportunidades para América Latina y el Caribe. Recuperado de http:// www.parlamericas.org/uploads/documents/ Primer_on_Climate_Financing_spa.pdf

PNUD (2016). Desde los ODM hasta el desarrollo sostenible para todos. Nueva York, Estados Unidos: Programa de las Naciones Unidas para el Desarrollo. Recuperado de http://www.undp. org/content/dam/undp/library/SDGs/Spanish/ ES_f_UNDP_MDGs-to-SDGs_web.pdf

PNUD (2019). Informe de los Objetivos de Desarrollo Sostenible 2019. Nueva York, Estados Unidos: Programa de las Naciones Unidas. Recuperado de https://unstats.un.org/sdgs/ report/2019/The-Sustainable-DevelopmentGoals-Report-2019_Spanish.pdf 
PNUMA (s.f.). Programa de las Naciones Unidas para el Medio Ambiente. Consultado en https:// www.un.org/ruleoflaw/es/un-and-the-rule-oflaw/united-nations-environment-programme/

PNUMA(2018).Informesobrela BrechadeEmisiones 2018. Resumen Ejecutivo. Publicaciones de las Naciones Unidas. Recuperado de https://wedocs. unep.org/bitstream/handle/20.500.11822/26879/ EGR2018_ESSP.pdf?sequence=19

Programa para el Medio Ambiente (s.f.). Financiación $y$ alianzas. Consultado en https://www.unenvironment.org/es/sobre-elprograma-de-la-onu-para-el-medio-ambiente/ financiacion-y-alianzas

Samaniego, J. y otros. (2019). Panorama de las contribuciones determinadas a nivel nacional en América Latina y el Caribe, 2019: Avances para el cumplimiento del Acuerdo de París. Santiago de Chile, Chile: Publicaciones de las Naciones Unidas. Recuperado de https://repositorio. cepal.org/bitstream/handle/11362/44974/1/ S1900855_es.pdf

Scardamaglia, V. (2019). Desafíos del financiamiento para la adaptación al cambio climático en América Latina y el Caribe. Red Regional de Cambio Climático y Toma de Decisiones. Recuperado de http://www. cambioclimaticoydecisiones.org/wp-content/ uploads/2019/08/POLICY-BRIEF_-DesafiosFinanciamiento_Scardamaglia_2019-.pdf
SICA (2010). Estrategia Regional de Cambio Climático. Recuperado de http:// www.sica.int/busqueda/busqueda_archivo. aspx?Archivo=info_55238_1_29112010.pdf

SICA (11 de mayo de 2018). SICA inicia Actualización de su Estrategia Regional de Cambio Climático. Recuperado de https://reliefweb.int/ report/el-salvador/sica-inicia-actualizaci-n-desu-estrategia-regional-de-cambio-clim-tico

SICA (2019). Estrategia Regional de Cambio Climático (ERCC) Actualizada. Recuperado de https://www.sica.int/documentos/estrategiaregional-de-cambio-climatico-ercc-actualizadaoctubre-2019_1_120055.html

Silva, A. y Mata de Grossi, M. (2005). La llamada Revolución Industrial. Caracas, Venezuela: Universidad Católica Andrés Bello. Consultado en https://books.google.com.sv/ books? id=YmbEneoFEI0C \&printsec $=$ front cover \&dq=\%22 revoluci $\%$ C3\%B3n +industrial\%22\&hl=es\&sa=X\&ved=0ahUKEwihiPWb4afpAhUDhOAKHSMLA_AQ6AEILTA$\mathrm{B} \# \mathrm{v}=$ onepage \&q $=\% 22$ revoluci $\% \mathrm{C} 3 \% \mathrm{~B} 3 \mathrm{n} \% 20$ industrial $\% 22 \& f=$ false

Vengoechea, A. (2012). Las cumbres de las Naciones Unidas sobre Cambio Climático. Colombia: Proyecto Energía y Clima de la Fundación Friedrich Ebert - FES. Recuperado de https://library.fes.de/pdf-files/bueros/laenergiayclima/09155.pdf 